\title{
Dramatic effect of fluid chemistry on cornstarch suspensions: Linking particle interactions to macroscopic rheology
}

\author{
Loreto Oyarte Gálvez, ${ }^{1}$ Sissi de Beer, ${ }^{2}$ Devaraj van der Meer, ${ }^{1}$ and Adeline Pons ${ }^{1, *}$ \\ ${ }^{1}$ Physics of Fluids Group, University of Twente, P.O. Box 217, NL-7500 AE Enschede, The Netherlands \\ ${ }^{2}$ Materials Science and Technology of Polymers, University of Twente, P.O. Box 217, NL-7500 AE Enschede, The Netherlands
}

(Received 17 November 2016; published 31 March 2017)

\begin{abstract}
Suspensions of cornstarch in water exhibit strong dynamic shear thickening. We show that partly replacing water with ethanol strongly alters the suspension rheology. We perform steady and nonsteady rheology measurements combined with atomic force microscopy to investigate the role of fluid chemistry on the macroscopic rheology of the suspensions and its link with the interactions between cornstarch grains. Upon increasing the ethanol content, the suspension goes through a yield-stress fluid state and ultimately becomes a shear-thinning fluid. On the cornstarch grain scale, atomic force microscopy measurements reveal the presence of polymers on the cornstarch surface, which exhibit a cosolvency effect. At intermediate ethanol content, a maximum of polymer solubility induces high microscopic adhesion which we relate to the macroscopic yield stress.
\end{abstract}

DOI: 10.1103/PhysRevE.95.030602

Suspensions are mixtures of undissolved particles in a liquid. They are literally found all around us: mud, paints, pastes, and blood [1]. The viscosity of a dense suspension can vary by orders of magnitude in a small shear rate interval [2]. Subjected to an increasing shear rate, dense suspensions first tend to become less viscous (shear thinning) and then more viscous (shear thickening). The viscosity of some suspensions, especially non-Brownian ones, may increase so much that they effectively become solid [3]. Although standard rheology measurements provide a great tool to study this phenomenon (see, e.g. Refs. [4,5]), they are mainly limited to steady-state conditions. Many studies point out that dense suspensions exhibit remarkable dynamic phenomena emerging under nonsteady-shear conditions: stable holes in thin vibrated layers [6], nonmonotonic settling [7], dynamic compaction front [8], or fracturing [3]. Oscillatory rheology helps to describe some of these dynamic behaviors [9], but remains limited to constant volume conditions.

Dynamic shear thickening has been widely investigated [10], but its physical origin remains an active debate. Although several parameters seem to contribute to it (e.g., particle size [11], shape [12], or roughness [13]), it has become increasingly clear that frictional and noncontact interactions between particles play a key role $[14,15]$. Such interactions are easily modified in numerical simulations, but present a real challenge in experiments. Consequently, only few experimental studies address the role of particle-particle interactions in dense suspension rheology (see, e.g., Refs. [5,16]), however lacking a systematic variation of these interactions. Moreover, direct measurements of these interactions in relation to the rheology are also lacking so far.

Here, we directly probe the microscopic interactions between individual particles and explore their link with the macroscopic rheology for dense cornstarch (CS) suspensions. The archetypical suspension of CS grains in water exhibits a strong dynamic shear thickening [3,6-8]. Interestingly, Taylor [17] shows that replacing water with polypropylene glycol

\footnotetext{
*adeline.pons@normalesup.org
}

in CS suspensions completely suppresses its shear-thickening nature and modifies its dielectric properties, reminiscent of observations in thermal suspensions $[18,19]$. Consequently, we tune particle interactions using suspending fluids with different chemical but similar physical properties (density, viscosity, etc.). Specifically, we systemically study water-ethanol mixtures in different proportions combining three different techniques: (1) non-steady-state rheology obtained from a sphere settling dynamics, (2) classical steady-state rheology, and (3) atomic force microscopy (AFM) to probe particle interactions. Both rheology techniques show that the typical shear-thickening behavior observed for pure water turns into a low viscosity shear thinning for pure ethanol, passing through a yield-stress-fluid state for intermediate mixtures. Furthermore, for water-based suspensions, shear-thickening and dynamic behaviors are observed, respectively in classical rheology and in settling experiments, at similar shear rate ranges. We relate this to AFM measurements showing that particle interactions vary as the fluid is changed. Our results indicate that CS grains are covered by chemical agents behaving similarly to what was recently observed for polymer brushes [20] exhibiting a cosolvency effect [21,22]. These dangling polymers may be at the origin of the peculiar rheology in water and also of the rheology changes with fluid as observed for colloidal suspensions (see, e.g., Refs. [1,23]).

Suspensions. The suspensions are mixtures of CS particles in water-ethanol solutions. CS particles have irregular shapes and diameters ranging from 5 to $20 \mu \mathrm{m}$. Freshly opened, $250 \mathrm{~g}$ sealed boxes of additive-free cooking CS were used. The density of CS from several boxes was determined by pycnometry: $\rho_{\mathrm{CS}} \sim 1542 \pm 15 \mathrm{~kg} \mathrm{~m}^{-3}$. The volume fraction of the suspensions is kept constant in this study: $\Phi_{\mathrm{CS}}^{v}=40 \%$. Although the true volume fraction might differ from $40 \%$, due to, e.g., particle porosity and moisture contents [24], our protocol ensures its reproducibility. The suspending fluid consists of a mixture of demineralized water and ethanol (99.8\%) from Atlas \& Assink Chemie. We vary the mass fraction of the solution $\Phi_{\mathrm{EtOH}}^{m}$ from $0 \%$ (pure water) to $100 \%$ (pure ethanol). The suspending fluids are prepared 1 day before the experiment, ensuring good mixing and cooling down. 

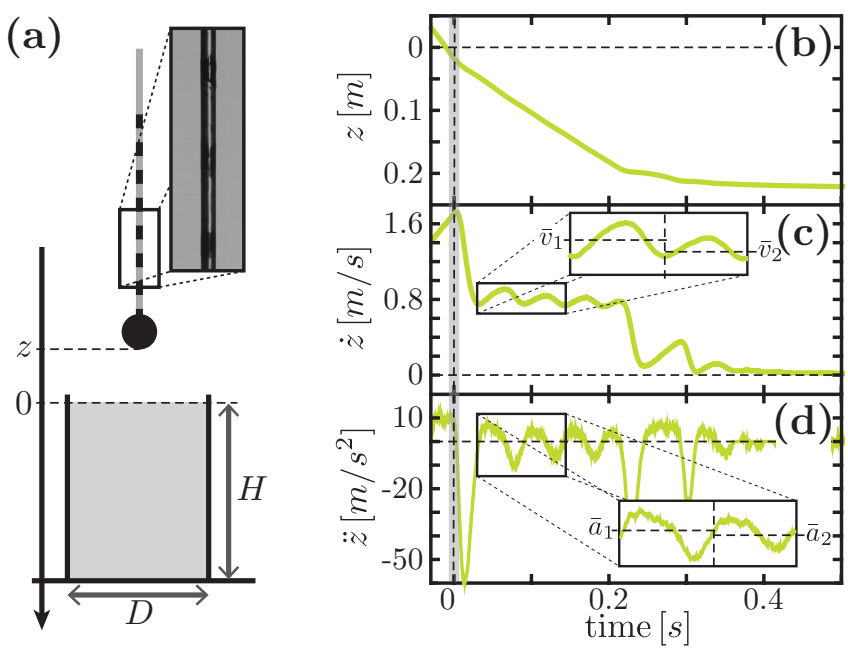

FIG. 1. (a) Experimental setup schematic. (b)-(d) Typical time evolution of vertical position $z$, velocity $\dot{z}$, and acceleration $\ddot{z}$ of the sphere for $\Phi_{\mathrm{EtOH}}^{m}=10 \%$ and $H_{\text {fall }}=15 \mathrm{~cm}$. The insets in (c) and (d) show two zoomed-in oscillations with their respective mean velocities $\left(\bar{v}_{1}, \bar{v}_{2}\right)$ and mean accelerations $\left(\bar{a}_{1}, \bar{a}_{2}\right)$.

Non-steady-state rheology. The experimental setup, shown in Fig. 1(a), consists of a cylindrical container (diameter $D=19.5 \mathrm{~cm}$, height $H=25 \mathrm{~cm}$ ) filled with the suspension into which we drop a sphere (mass $m_{s}=248 \mathrm{~g}$, radius $R_{s}=1.54 \mathrm{~cm}$ ). The release height $H_{\text {fall }}$ varies between $-2 R_{s}$ (sphere starting immersed) and $30 \mathrm{~cm}$. In order to follow the settling dynamics, a thin and rigid metal wire with tracers is attached to the top of the sphere. The mass of the wire $(\sim 1 \mathrm{~g})$ and its resulting buoyancy force can be neglected compared to the sphere. We follow the tracers at a frame rate between 500 and $5000 \mathrm{~Hz}$ using a high speed camera (SA7, Photron). Correlating successive images, we determine the sphere vertical position $z$, velocity $\dot{z}$, and acceleration $\ddot{z}$ during its settling.

Figures 1(b)-1(d) show the time evolution of $z, \dot{z}$, and $\ddot{z}$ for $\Phi_{\mathrm{EtOH}}^{m}=10 \%$ and $H_{\text {fall }}=15 \mathrm{~cm}$. As previously observed for a CS suspension using pure water [7,25], after a rapid slowing down due to the impact (gray vertical line), $\dot{z}$ oscillates around a terminal velocity. For each oscillation we define its mean velocity $\bar{v}_{i}$ and mean acceleration $\bar{a}_{i}$ [insets of Figs. 1(c) and 1(d)]. When approaching the bottom, the sphere comes to a sudden full stop at $\sim 20 \mathrm{~mm}$ above the bottom. Then, it reaccelerates until it stops again. This repetitive stop-and-go behavior is due to successive jamming and unjamming of the granular skeleton between the intruder and the bottom [7].

When varying $\Phi_{\mathrm{EtOH}}^{m}$, we observe a continuous change in settling dynamics (Fig. 2). As $\Phi_{\mathrm{EtOH}}^{m}$ is increased up to $\sim 50 \%$, the suspension viscosity rises (the average settling velocity decreases) and the oscillations disappear [Fig. 2(a)]. Beyond $\Phi_{\mathrm{EtOH}}^{m} \sim 70 \%$, the viscosity becomes so small that the sphere bounces on the container bottom [Fig. 2(b)].

We can distinguish three typical behaviors, illustrated in Figs. 3(a)-3(f). Figures 3(a)-3(c) show the influence of the initial velocity for $\Phi_{\mathrm{EtOH}}^{m}=0 \%, 50 \%$, and $100 \%$ on the settling dynamics and Figs. 3(d)-3(f) show the drag force $F_{D}$ as a function of $\dot{z} \cdot F_{D}$ is derived from $\ddot{z}$ using the force balance on
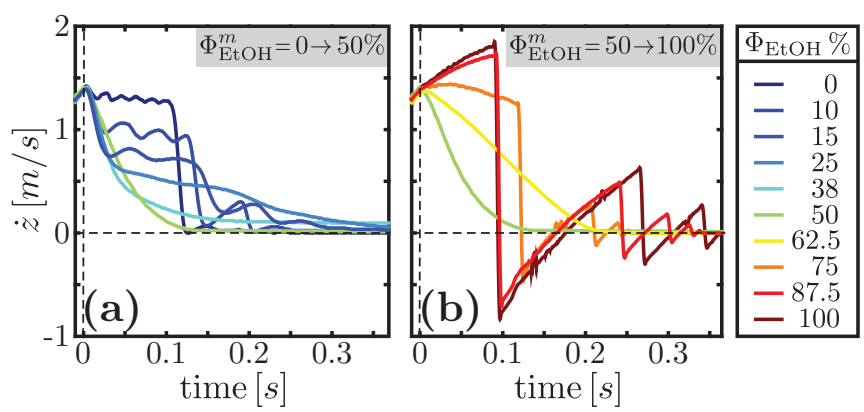

FIG. 2. Sphere velocity $\dot{z}$ as a function of time for various $\Phi_{\mathrm{EtOH}}^{m}$ and $H_{\text {fall }}=10 \mathrm{~cm}$.

the sphere,

$$
F_{D}=m_{s}(\ddot{z}-g)+\frac{4}{3} \pi R_{s}^{3} \rho_{\text {susp }} g,
$$

in which $\rho_{\text {susp }}$ is the suspension density and $g$ is the gravitational constant.

Up to $\Phi_{\mathrm{EtOH}}^{m} \sim 20 \%-25 \%$, the dynamics is similar to that of pure water [Figs. 3(a) and 3(d)]. Interestingly, the terminal velocity decreases with increasing $\Phi_{\mathrm{EtOH}}^{m}$ but is independent of the initial velocity $V_{0}$. For low $V_{0}$, the sphere accelerates towards this terminal velocity. In contrast, for higher $V_{0}$, velocity decreases with oscillations reaching the same terminal velocity. $F_{D}$ increases linearly with $\dot{z}$ up to a critical velocity ( $\sim 0.9 \mathrm{~m} / \mathrm{s}$ for pure water). Above this critical velocity, oscillations are observed and the period averages $\bar{F}_{D}$ vs $\bar{v}$ [gray squares in Fig. 3(d)] collapse onto a unique curve whose slope seems to slightly increase with velocity, corresponding to an increased viscosity which is typical of a shear-thickening fluid.

For intermediate $\Phi_{\mathrm{EtOH}}^{m}$ (between $\sim 20 \%-25 \%$ and $\sim 70 \%$ ), the sphere decelerates very rapidly after penetrating into the suspension. It then sinks at a constant velocity close to zero. Consistently, Fig. 3(e) shows that $F_{D}$ equals the sphere weight in the limit of zero velocity. These two behaviors are typical of a yield-stress fluid when the density of the object is slightly above the critical density relative to the yield stress [26].

Finally, for $\Phi_{\text {EtOH }}^{m} \gtrsim 70 \%$ [Figs. 3(c) and 3(f)], the sphere encounters a small drag resistance and bounces on the container bottom several times. Taking into account the noisy nature of the measurement, drag force curves for the different $H_{\text {fall }}$ collapse onto a single curve, regardless of the velocity sign.

Classical rheology. We use an MCR 502 rheometer (Anton Paar) with a concentric cylinder geometry. All measurements are repeated at least three times. Figures 3(g)-3(i) present the flow curves obtained from these rheological measurements (blue circles). They are compared to the dynamical behavior of the suspensions obtained from settling experiments (orange diamonds). To do such a comparison, we define an apparent viscosity $\eta^{*}=F_{D} /\left(6 \pi R_{s} \dot{z}\right)$ and a characteristic shear rate $\dot{\gamma}^{*}=\dot{z} / R_{s}$. Although Stokes' law is not applicable, it provides a reasonable estimation of the dynamic viscosity.

The flow curves obtained from steady-state classical rheology and from our dynamic system present a convincing qualitative agreement, although the numerical values are different, probably due to approximations (Stokes' law) or geometrical factors. For $\Phi_{\text {EtOH }}^{m}$ above $\sim 20 \%-25 \%$ these suspensions all 

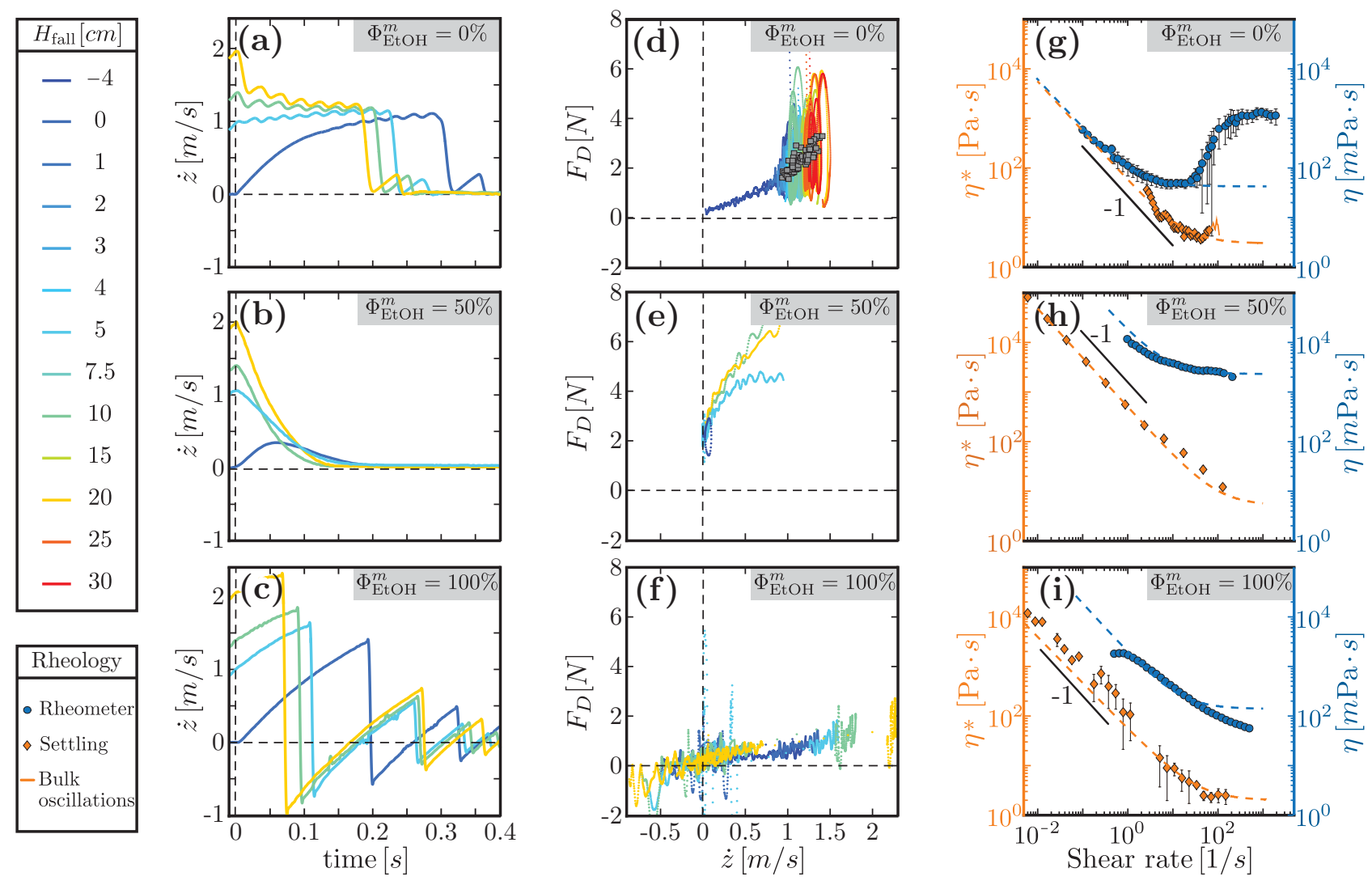

FIG. 3. Non-steady-state and classical rheology as a function of $\Phi_{\mathrm{EtOH}}^{m}$ : (a)-(c) Sphere velocity $\dot{z}$ as a function of time for various $H_{\text {fall }}$. (d)-(f) Drag force $F_{D}$ encountered by the sphere as a function of its velocity for the same and additional experiments. The gray squares in (d) show the mean drag force as a function of mean velocity $\bar{v}$ during oscillations. (g)-(i) Flow curves from classical rheological measurements (blue circles) and apparent flow curves obtained from settling experiments (orange diamonds). The dashed lines in (g)-(i) are the best fits of the data with the Bingham model and the orange line in $(\mathrm{g})$ corresponds to the bulk oscillations' mean behavior.

present a yield stress and can be described by a simple Bingham equation, $\eta_{B}=\eta_{\mathrm{pl}}+\sigma_{Y} / \dot{\gamma}$, in which $\eta_{\mathrm{pl}}$ is the plastic viscosity and $\sigma_{Y}$ the yield stress. This is consistent with earlier observations in CS suspensions with \{water-polypropylene glycol\} solutions [17]. For lower $\Phi_{\mathrm{EtOH}}^{m}$, a Bingham equation can also approximate the flow curves for low shear rates. The values of $\sigma_{Y}$ as a function of $\Phi_{\mathrm{EtOH}}^{m}$ from both rheological measurements are shown in Fig. 4(e). For both methods, $\sigma_{Y}$ reaches a maximum value for intermediate $\Phi_{\mathrm{EtOH}}^{m}$ (between $\sim 25 \%$ and $\sim 70 \%$ ). Finally, for low $\Phi_{\mathrm{EtOH}}^{m}$ and high shear rates the steady-state rheology exhibits a strong shear thickening which corresponds to the conditions in which bulk oscillations are observed during the settling experiments.

Particle-particle interactions. We probe the interactions between CS particles using atomic force microscopy (AFM) by attaching single CS grains to tipless cantilevers (see the Appendix for experimental details). We measure the force curves [Fig. 4(a)] while approaching and retracting this CS grain to other CS grains glued on the surface of a stainless steel disk in different water-EtOH solutions. From the force curves, we measure the adhesion force $F_{\text {adh }}$ between individual CS grains and their apparent Young's modulus $E^{*}$. We also estimate an interaction length $L_{\text {int }}$ corresponding to the separation at which grains start to feel each other. Details on the analysis procedure can be found in the Appendix.
Figure 4(a) shows a typical force curve obtained in water, representing the force between the $\mathrm{CS}$ particle on the cantilever and one on the surface when approaching (blue) and retracting (red). On the retracting curve we observe sharp steps called pulling events. These events are signatures of high density dangling polymers disentangling in mediocre solvents [20]. This is a plausible explanation as CS is made of alternating semicrystalline and amorphous layers of the biopolymers amylose and amylopectin [27], being respectively slightly and mostly soluble in cold water [28] but less and less soluble as ethanol is added to the solvent (see, e.g., Refs. [29,30]) until being insoluble in ethanol [31]. Figure 4(b) shows the percentage of measurements with pulling events as a function of $\Phi_{\mathrm{EtOH}}^{m}$. We observe them for all ethanol concentrations with a minimum for ethanol, logical with amylose and amylopectin solubilities, and a maximum for intermediate concentrations, which indicates a cosolvency effect which is a solubility maximum at intermediate $\Phi_{\mathrm{EtOH}}^{m}[20-22]$.

Figure 4(c) shows the apparent elastic modulus $E^{*}$ of one grain (blue) and the interaction length $L_{\text {int }}$ between two grains (orange). As the grains are not spherical and have sizes ranging from 5 to $20 \mu \mathrm{m}$, contact areas and curvature radii are difficult to assess, which are responsible for the large error bars. Therefore, we should not attach too much significance to the absolute values, but information from the data comparison for the different values of $\Phi_{\mathrm{EtOH}}^{m}$ is to be trusted. Thus we 

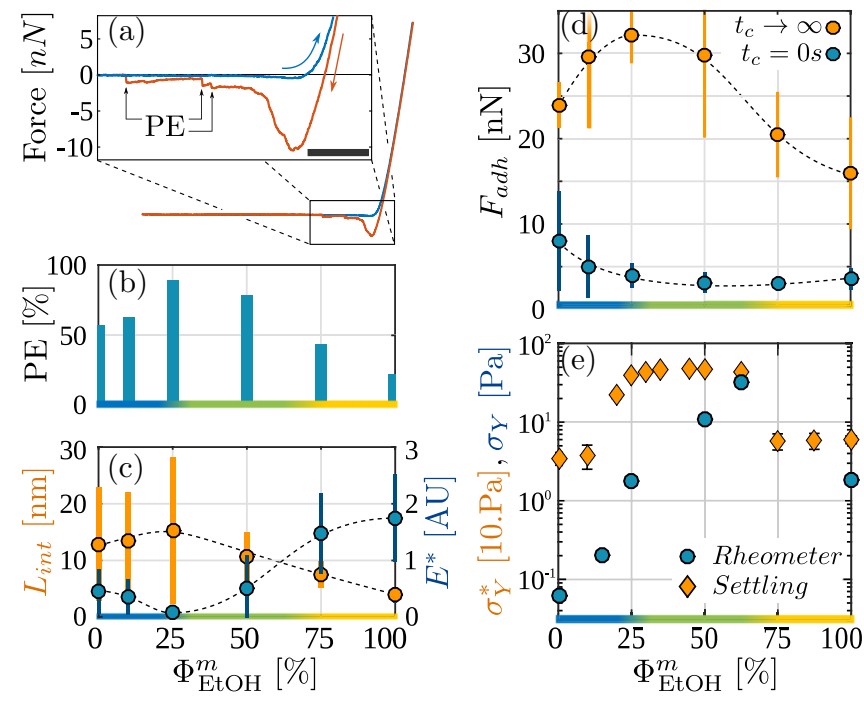

FIG. 4. Particle-particle interaction properties as a function of $\Phi_{\mathrm{EtOH}}^{m}$ : (a) Typical force curve in water. The inset and arrows show the presence of pulling events (PEs) while retracting (red curve). The black bar corresponds to a vertical displacement of $30 \mathrm{~nm}$. (b) Percentage of force curves exhibiting PEs. (c) Apparent Young's modulus $E^{*}$ of an individual CS grain and interaction length $L_{\text {int }}$ between CS grains. (d) Adherence force $F_{\text {adh }}$ between two CS grains for a contact time of $0 \mathrm{~s}$ (blue) and in the limit of infinite contact time (orange). (e) Yield stress measured from classical rheology (orange) and settling experiments (blue). The dashed lines are just guides to the eye. The color scales on the horizontal axis stand for the three typical behaviors: shear thickening and bulk oscillation (blue), yield-stress fluid (green), and shear-thinning fluid (yellow).

observe that the apparent particle softness and the interaction length vary with $\Phi_{\mathrm{EtOH}}^{m}$ which we interpret as a result of the cosolvency effect: $\Phi_{\mathrm{EtOH}}^{m} \approx 25 \%$ appears to be the best solvent, which is consistent with more pulling events being observed for this concentration. Indeed, a better solvent allows for deeper interdigitation of the polymers in opposing grains. Although $L_{\text {int }}$ varies inversely to $E^{*}$, from our data it is not possible to determine the origin of the repulsive force before elastic contact: It could be either interdigitation of the polymers or some form of noncontact repulsion, such as static charges.

After the approach, it is possible to keep two CS grains in contact for a given contact time $t_{c}$ before retracting. By doing so, we can investigate the effect of the contact time on the adherence force $F_{\text {adh }}$. Figure 4(d) shows the evolution of $F_{\text {adh }}$ as a function of $\Phi_{\mathrm{EtOH}}^{m}$ for zero contact time $F_{\mathrm{adh}}^{0}$ (blue) and in the limit of infinite contact time $F_{\text {adh }}^{\infty}$ (orange). For all $\Phi_{\mathrm{EtOH}}^{m}, F_{\text {adh }}$ increases with $t_{c}$ following an exponential decay characterized by a decay time $\tau$ (see the Appendix for details), which is again consistent with our interpretation of free dangling polymers interpenetrating with time. We observe that $F_{\text {adh }}^{0}$ is maximal in pure water whereas $F_{\text {adh }}^{\infty}$ exhibits a maximum for $\Phi_{\mathrm{EtOH}}^{m}=25 \%$. We attribute the latter to the larger effective interaction area due to particle softness and polymer interpenetration.

These results are consistent with the macroscopic rheology. Indeed, $F_{\text {adh }}^{\infty}$ must be related to the suspension behavior at small shear rate, i.e., the yield stress $\sigma_{Y}$, which we obtain from the Bingham fits to the flow curves of Fig. 3. Although slightly shifted, $F_{\text {adh }}^{\infty}$ shows similar variations as the yield stress extracted from rheology experiments [Fig. 4(e)]. Moreover, the shear-thinning part of the flow curves observed for all $\Phi_{\mathrm{EtOH}}^{m}$ is also consistent with an increase of $F_{\text {adh }}$ with $t_{c}$. On the other hand, one could expect that the suspension behavior at high shear rates could be related to $F_{\text {adh }}^{0}$ and $\tau$. But the present measurements do not show any quantitative indication of that, although $\tau$ does vary with $\Phi_{\mathrm{EtOH}}^{m}$. Namely, $\tau$ is minimal for pure water $\left(\tau_{\min }=0.5 \pm 0.1 \mathrm{~s}\right)$ and maximal for intermediate concentrations $\left(\tau_{\min }=2.0 \pm 0.7 \mathrm{~s}\right.$ ) (see the Appendix). Therefore, friction measurements as described in Ref. [32] could provide additional insights [13,33], although with interpretation difficulties due to CS particle irregularity and roughness.

Summary. In this Rapid Communication we show that by gradually replacing the suspending fluid of the well-known suspension of cornstarch and water with ethanol, the familiar shear-thickening behavior completely disappears. Going from pure water to pure ethanol, the suspension behavior changes continuously with ethanol concentration from dynamic shear thickening for pure water to low viscosity shear thinning for pure ethanol, passing through a yield-stress-fluid phase for intermediate mixtures. A comparison of classical (steadystate) and non-steady-state settling rheology shows qualitative agreement. More specifically, it shows that flow conditions for which shear thickening is observed in classical rheology measurements correspond to the conditions for which bulk oscillations are observed in non-steady-state experiments.

These behaviors are related to the interactions between CS grains in the different suspending fluids measured using atomic force microscopy. We first present evidence that CS grains are covered by free dangling polymers behaving as polymer brushes. Then, the variation of the adherence force with the suspending fluid is shown to be consistent with the yield stress observed in macroscopic rheology. This indicates that the macroscopic behavior is closely linked to the details of the particle-particle interactions. It appears that the presence of dangling polymers may not only be at the origin of the strikingly different behaviors observed while changing the suspending fluid, but also of the peculiar dynamic behavior of suspensions of $\mathrm{CS}$ in water. In order to validate this hypothesis, it is essential to perform additional research on better controlled systems such as suspensions of spherical particles functionalized with known polymer brushes.

Acknowledgments. The authors thank H. Gojzewski for preparing the cantilevers. This work is part of a research program of the Foundation for Fundamental Research on Matter (FOM), which is financially supported by the Netherlands Organisation for Scientific Research (NWO). L.O.G. is supported by Becas Chile-CONICYT and A.P. by a FOM/v postdoctoral grant.

\section{APPENDIX: ATOMIC FORCE MICROSCOPY (AFM)}

\section{Experimental details}

We use a Bruker AFM (Multimode 8 with a Nanoscope V controller) using a JV vertical engage scanner and a Bruker glass liquid cell. Using a micromanipulator and UV curing 

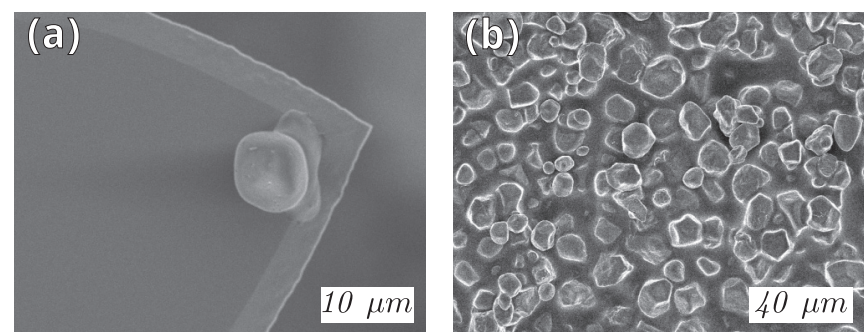

FIG. 5. Scanning electron microscopy (SEM) image of (a) a cornstarch grain glued to the end of a tipless AFM cantilever and (b) a sample surface covered with cornstarch grains.

glue (NOA 81) we attached a single cornstarch particle to the end of three tipless AFM cantilevers (TL-FM, sQube, Germany), with spring constants $3.24,2.93$, and $2.61 \mathrm{~N} \mathrm{~m}^{-1}$ and resonance frequencies of 85,75 , and $79 \mathrm{kHz}$, respectively [Fig. 5(a)]. The tested surfaces consist of stainless steel disks covered with cornstarch particles, attached using an epoxy two-component glue [Fig. 5(b)]. Force curves are measured while approaching and retracting the cornstarch grain to and from these surfaces in different water-EtOH solutions $\left(\Phi_{\mathrm{EtOH}}^{m}=0 \%, 10 \%, 25 \%, 50 \%, 75 \%\right.$, and $\left.100 \%\right)$ with a velocity of $0.77,1.44-2.88 \mu \mathrm{m} \mathrm{s}^{-1}$, for which the analysis shows no influence of the approaching and retracting velocity.

With the different cantilevers, we probe three positions on two different samples and compared them to reference force curve measurements on bare glue to ensure that we truly probe the CS-CS interactions. For each position, force curves are averaged over at least 50 measurements. Figure 4 shows averaged results for one cantilever and sample set. Other cantilevers and samples show similar trends.

\section{Force curve analysis}

The force measured upon close approach was fitted with the Hertzian contact model following the procedure presented in Refs. [34,35] to obtain $E^{*}$ [Fig. 4(c)] and the position at which the contact starts to become elastic. This point is considered as the contact point. As the cornstarch grains are not perfectly round and their radius not well defined, we only obtain an apparent modulus. Thus, absolute values have no concrete interpretation but can be compared for different suspending fluids. The maximum force reached is $60-80 \mathrm{nN}$.
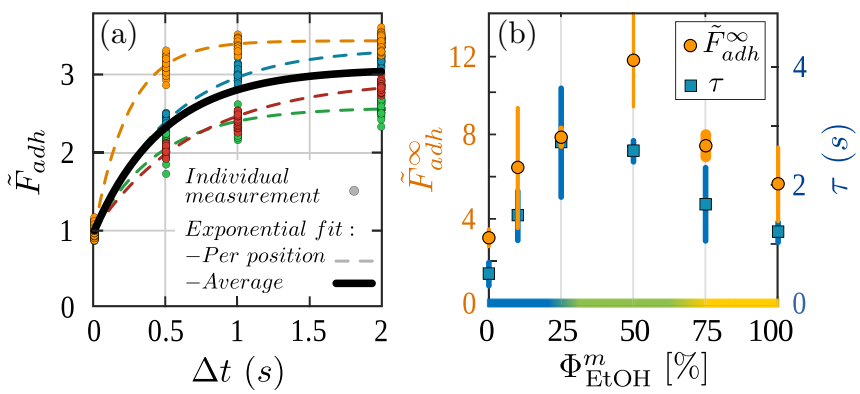

FIG. 6. (a) Variation of $\tilde{F}_{\text {adh }}$ as a function of waiting time. Colors stand for each probing position. Dashed lines correspond to the exponential fit for each position and the solid line is the average fit. (b) Average $\tilde{F}_{\text {adh }}^{\infty}$ and $\tau$ as a function of $\Phi_{\mathrm{EtOH}}^{m}$.

The adhesion $F_{\text {adh }}$ [Fig. 4(d)] is the force just before the cornstarch grains snap out of contact while retracting the cantilever.

\section{Effect of contact duration on the adhesion}

As the rheology of cornstarch suspensions is observed to strongly depend on the shear rate, we study the evolution of the adhesion force as a function of the contact duration between two grains. To do so, we approach the grain to the surface, keep the grains in contact during a waiting time $\Delta t$ ranging from 0 to $20 \mathrm{~s}$, and then retract. These measurements are performed in different water-EtOH solutions with a velocity of $1.44 \mu \mathrm{m} \mathrm{s}^{-1}$.

As the geometry of the contact may vary from one probing position to another, for each position we normalize the adhesion force by the one corresponding to zero waiting time:

$$
\tilde{F}_{\text {adh }}(\Delta t)=\frac{F_{\text {adh }}(\Delta t)}{F_{\text {adh }}(\Delta t=0 \mathrm{~s})} .
$$

Figure 6(a) shows the variation of the normalized adhesion force $\tilde{F}_{\text {adh }}$ as a function of the waiting time for $\Phi_{\mathrm{EtOH}}^{m}=0 \%$ and for each different probing position. The normalized adhesion force can be fitted by an exponential

$$
\tilde{F}_{\text {adh }}(\Delta t)=\tilde{F}_{\text {adh }}^{\infty}-\left(\tilde{F}_{\text {adh }}^{\infty}-1\right) \exp \left(-\frac{\Delta t}{\tau}\right) .
$$

$\tilde{F}_{\text {adh }}^{\infty}$ and $\tau$ are measured for each position and for each $\Phi_{\mathrm{EtOH}}^{m} \cdot$ The values shown in Figs. 4(d) and 6(b) correspond to the average over all positions for each $\Phi_{\mathrm{EtOH}}^{m}$.
[1] N. J. Wagner and J. F. Brady, Phys. Today 62(10), 27 (2009).

[2] H. A. Barnes, J. Rheol. 33, 329 (1989).

[3] M. Roché, E. Myftiu, M. C. Johnston, P. Kim, and H. A. Stone, Phys. Rev. Lett. 110, 148304 (2013).

[4] A. Fall, F. Bertrand, G. Ovarlez, and D. Bonn, J. Rheol. 56, 575 (2012).

[5] E. Brown, N. A. Forman, C. S. Orellana, H. Zhang, B. W. Maynor, D. E. Betts, J. M. DeSimone, and H. M. Jaeger, Nat. Mater. 9, 220 (2010).

[6] F. S. Merkt, R. D. Deegan, D. I. Goldman, E. C. Rericha, and H. L. Swinney, Phys. Rev. Lett. 92, 184501 (2004).

[7] S. von Kann, J. H. Snoeijer, D. Lohse, and D. van der Meer, Phys. Rev. E 84, 060401 (2011).
[8] S. R. Waitukaitis and H. M. Jaeger, Nature (London) 487, 205 (2012).

[9] R. D. Deegan, Phys. Rev. E 81, 036319 (2010).

[10] E. Brown and H. M. Jaeger, Rep. Prog. Phys. 77, 046602 (2014).

[11] B. M. Guy, M. Hermes, and W. C. K. Poon, Phys. Rev. Lett. 115, 088304 (2015).

[12] E. Brown, H. Zhang, N. A. Forman, B. W. Maynor, D. E. Betts, J. M. DeSimone, and H. M. Jaeger, Phys. Rev. E 84, 031408 (2011).

[13] L. C. Hsiao, S. Jamali, D. J. Beltran-Villegas, E. Glynos, P. F. Green, R. G. Larson, and M. J. Solomon, arXiv:1610.09314.

[14] R. Seto, R. Mari, J. F. Morris, and M. M. Denn, Phys. Rev. Lett. 111, 218301 (2013). 
[15] R. Mari, R. Seto, J. F. Morris, and M. M. Denn, Proc. Natl. Acad. Sci. USA 112, 15326 (2015).

[16] N. Y. C. Lin, B. M. Guy, M. Hermes, C. Ness, J. Sun, W. C. K. Poon, and I. Cohen, Phys. Rev. Lett. 115, 228304 (2015).

[17] S. E. Taylor, J. Dispersion Sci. Technol. 34, 887 (2013).

[18] G. V. Franks, Z. Zhou, N. J. Duin, and D. V. Boger, J. Rheol. 44, 759 (2000).

[19] C. D. Cwalina and N. J. Wagner, J. Rheol. 58, 949 (2014).

[20] Y. Yu, B. D. Kieviet, E. Kutnyanszky, G. J. Vancso, and S. de Beer, ACS Macro Lett. 4, 75 (2015).

[21] L. H. Cragg and H. Hammerschlag, Chem. Rev. 39, 79 (1946).

[22] D. Mukherji, C. M. Marques, T. Stuehn, and K. Kremer, arXiv:1609.09839.

[23] J. Mewis and G. Biebaut, J. Rheol. 45, 799 (2001).

[24] I. R. Peters and H. M. Jaeger, Soft Matter 10, 6564 (2014).

[25] S. von Kann, J. H. Snoeijer, and D. van der Meer, Phys. Rev. E 87, 042301 (2013).

[26] H. Tabuteau, P. Coussot, and J. R. de Bruyn, J. Rheol. 51125 (2007).
[27] A. Buléon, P. Colonna, V. Planchot, and S. Ball, Int. J. Biol. Macromol. 23, 85 (1998).

[28] M. M. Green, G. Blankenhorn, and H. Hart, J. Chem. Educ. 52, 729 (1975).

[29] A. J. Barrett, K. L. Barrett, and A. Khan, J. Macromol. Sci., Part A 35, 1603 (1998).

[30] M. E. Zakrzewska, E. Bogel-Łukasik, and R. Bogel-Łukasik, Energy Fuels 24, 737 (2010).

[31] C. Greenwood, in The Carbohydrates, 2nd ed., edited by W. Pigman and D. Horton (Academic Press, New York, 1970), pp. 471-513.

[32] N. Fernandez, J. Cayer-Barrioz, L. Isa, and N. D. Spencer, Langmuir 31, 8809 (2015).

[33] N. Fernandez, R. Mani, D. Rinaldi, D. Kadau, M. Mosquet, H. Lombois-Burger, J. Cayer-Barrioz, H. J. Herrmann, N. D. Spencer, and L. Isa, Phys. Rev. Lett. 111, 108301 (2013).

[34] D. C. Lin, E. K. Dimitriadis, and F. Horkay, J. Biomech. Eng. 129, 430 (2007).

[35] D. C. Lin, E. K. Dimitriadis, and F. Horkay, J. Biomech. Eng. 129, 904 (2007). 